\title{
Frequency Triplexer for Ultra-Wideband Systems (6-9 GHz)
}

Magnus Karlsson, Adriana Serban, Joakim Östh, Owais Owais and Shaofang Gong

\author{
Linköping University Post Print
}

\section{Tweet}

N.B.: When citing this work, cite the original article.

(C)2013 IEEE. Personal use of this material is permitted. However, permission to reprint/republish this material for advertising or promotional purposes or for creating new collective works for resale or redistribution to servers or lists, or to reuse any copyrighted component of this work in other works must be obtained from the IEEE.

Magnus Karlsson, Adriana Serban, Joakim Östh, Owais Owais and Shaofang Gong, Frequency Triplexer for Ultra-Wideband Systems (6-9 GHz), 2013, IEEE Transactions on Circuits and Systems Part 1: Regular Papers, (60), 3, 540-547.

http://dx.doi.org/10.1109/TCSI.2012.2215743

Postprint available at: Linköping University Electronic Press http://urn.kb.se/resolve?urn=urn:nbn:se:liu:diva-90756 


\title{
Frequency Triplexer for Ultra-wideband Systems (6-9 GHz)
}

\author{
Magnus Karlsson, Adriana Serban, Joakim Östh, Owais, and Shaofang Gong, Member, IEEE
}

\begin{abstract}
A triplexer for ultra-wideband radio implemented entirely with distributed microstrips is presented. The triplexer is composed of a triplex junction network and three bandpass filters. The circuit is integrated in a flex-rigid printed circuit board. Three flat $1 \mathrm{GHz}$ sub-bands in the frequency band 6-9 $\mathrm{GHz}$ have been achieved. The group delay variation within each $1 \mathrm{GHz}$ sub-band was measured to be less than $0.4 \mathrm{~ns}$. A good agreement between simulation and measurement was obtained, e.g., less than $0.3 \mathrm{~dB}$ difference in the forward transmission in the majority of the frequency band.
\end{abstract}

Index Terms-Bandpass filter, Broadside-coupled, Edgecoupled, Frequency multiplexing, Triplexer, UWB

\section{INTRODUCTION}

$\mathrm{T}$ The principle of frequency multiplexing, i.e., to combine the spectra from several ports into one wide spectrum has previous been proposed in a number of ways [1]-[12]. Suggestions about how to implement multiplexing or preselecting techniques in ultra-wideband (UWB) systems have been made in [13]-[24]. The interest for high speed and short range wireless-communication has been one of the major driving forces behind the UWB development [25]-[28]. In earlier time the band 3.1-4.8 GHz, was the primary frequencyband used for UWB. Later on legal respiratory around the world has adopted additional constraints on the use of the UWB frequency-band [29]-[34]. As a result the frequencybands 6-8.5, 6-9, and 6-10.6 GHz have lately received an increased attention [30] and [31]. We have recently proposed a frequency multiplexing technique in our study on ultrawideband antennas [20], transceivers [21] and [22]. A study of a multiplexer using planar microstrips was conducted in [23].

Frequency multiplexing is a technique that has been studied for decades and many implementations have been demonstrated using various techniques [1], [23]. For instance, references [2]-[3] present a generalized form of the manifold theory. Reference [4] presents an optimization algorithm for waveguide multiplexers. Reference [7] demonstrates methods for reducing the need of experimental correction when using computer-aided design. Reference [8] presents a lumped element and manifold microwave multiplexer using the hightemperature superconductor technology. Multiplexer design

Manuscript received Oct. 3, 2011; revised Jan. 12, 2012. Sony Mobile Communications AB, Ericsson AB and Vinnova in Sweden are acknowledged for financial support of this work.

Magnus Karlsson; email: magka@itn.liu.se, et al., are with Linköping University, Sweden. using printed circuit boards typically involves some lumped components, as in [10] both a duplexer and a triplexer are presented. Paper [13] proposes a stepped-impedance multiplexer for UWB and wireless local area network (WLAN) coexistence. In [14], a narrow-band four-channel multiplexer using open loop resonators for multi-band on-off keying UWB is demonstrated. Papers [15]-[16] show two channel-select multiplexers intended for UWB local oscillator signal selection, i.e., only one sub-band is active at the time. Some recent achievements are for instance a multiplexer with four narrow channels that are well separated (large guard band between the channels) is presented in [18]. Moreover, enhancement techniques in waveguide multiplexers due to optimization of the distance between channel filters placement [19]. Microstrip and stripline filters have been presented in many shapes and realized with various resonators, e.g., edge coupled and broadside coupled microstrips [23], [35] and [36]. One of the most common applications for a multiplexing circuit is a duplexer connecting $\mathrm{Rx}$ and $\mathrm{Tx}$ to the same antenna [37]. To achieve a good isolation between $\mathrm{Rx}$ and $\mathrm{Tx}$ can be achieved with sufficient channel spacing, as in the dual band impulse UWB radio proposed in [38]. A system with two channels centered at 3.5 and $4.5 \mathrm{GHz}$, respectively [38]. It is clear that also state of the art communication systems benefits from multi channel capability [37]-[39]. Hence, with an adequate triplexer a system capable of handling three high performance well isolated channels is possible.

Our previous paper [20] proposes antenna parallelism using frequency multiplexing. Our second paper [21] on the subject suggests using the multiplexer from [20] as pre-select filter for a UWB low-noise amplifier (3.1-4.8 GHz). In our third paper [22] the maximum theoretical data-rate limits of the proposed 11 sub-channel system using multiplexing techniques to combine the channels are studied. In our fourth paper [23] a multiplexer solution with three $500 \mathrm{MHz}$ sub-bands implemented on a conventional printed circuit board was presented. Moreover, the idea of using the triplexer presented in [23] for parallel UWB channels was discussed in [24]. The triplexer in this paper is intended for 6-9 GHz UWB using a flex-rigid printed circuit board. Flexible materials have been used in wired interconnects, i.e., mainly in high speed interconnects in the Gbps domain [39]. Using the flex-rigid technology an antenna can easily be integrated in the flexible part of the printed circuit board while other components can be integrated in the rigid part [40]. One of the uniqueness's 
with the design presented in this paper is that the triplexer is fully integrated in the "rigid part" of the flex-rigid printed circuit board using microstrips, resulting in a high performance and flexible solution but at low cost. Moreover, a detailed substrate stack was used to obtain good agreement between simulated and measured results. Most frequency multiplexing techniques published in the literature have used either waveguides or hybrids, having inherent difficulty for system integration and miniaturization. The flex-rigid substrate used in this study allows full integration of the radio front-end including the antenna [40]. One of the major challenges dealt with in this paper is that the sub-bands are side by side, i.e., virtually no guard-band. A typical guardband normally has a relative bandwidth of $10 \%$ [23]. However, if such a large guard-band were to be used too much frequency capacity would be lost. Hence, if only considering the two guard-bands in between the three subbands $1.5 \mathrm{GHz}$ out of the $3 \mathrm{GHz}$ would not be useable for data transmission.

\section{OVERVIEW OF THE SYSTEM}

All prototypes were manufactured using a flex-rigid printed circuit board with four metal-layer in the rigid part. The base substrate material is Pyralux polyimide from Dupont, processed together with a prepregs, as shown in Fig. 1. Prepreg is a sheet material (e.g., glass fabric) impregnated with a resin cured to an intermediate stage, ready for one stage printed circuit board bonding.

Table 1. Printed circuit board parameters

\begin{tabular}{ll}
\hline Parameter (Polyimide) & Dimension \\
\hline Dielectric constant & $3.40 \pm 0.05$ \\
Dissipation factor & 0.004 \\
\hline Parameter (Adhesive) & Dimension \\
\hline Dielectric constant & $3.60 \pm 0.10$ \\
Dissipation factor & 0.020 \\
\hline Parameter (Metal, common) & Dimension \\
\hline Metal thickness, layer 1, 4 & $0.038 \mathrm{~mm}$ \\
Metal thickness, layer 2, 3 & $0.018 \mathrm{~mm}$ \\
Metal conductivity & $5.8 \times 10^{7} \mathrm{~S} / \mathrm{m}$ (Copper) \\
Surface roughness & $0.001 \mathrm{~mm}$ \\
\hline
\end{tabular}

Table 1 lists the printed circuit board parameters, with the stack of the printed circuit board layers shown in Fig. 1. Metal layers 1 and 4 are thicker than metal layers 2 and 3 because the surface layers are plated, when plating the VIAs of the printed circuit board. Only drilled through hole VIAs were used. No signal VIAs are needed in the design due to the utilization of broadside coupling. However, a number of ground VIAs were used to ensure a low impedance ground connection throughout the desired frequency band (Clearly seen later in Fig. 2, in section II-A).
Table 2. Printed circuit board build-up

\begin{tabular}{ll}
\hline Rigid 1 $(0.1524 \mathrm{~mm})$ & Dimension \\
\hline Metal 1 & $0.0380 \mathrm{~mm}$ \\
Adhesive & $0.0254 \mathrm{~mm}$ \\
Polyimide & $0.0508 \mathrm{~mm}$ \\
Adhesive & $0.0254 \mathrm{~mm}$ (Bonds rigid-flex) \\
\hline Coating 1 $(0.0508 \mathrm{~mm})$ & Dimension \\
\hline Polyimide & $0.0254 \mathrm{~mm}$ \\
Adhesive & $0.0254 \mathrm{~mm}$ \\
\hline Flex $(0.1270 \mathrm{~mm})$ & Dimension \\
\hline Metal 2 & $0.0180 \mathrm{~mm}$ \\
Polyimide & $0.0508 \mathrm{~mm}$ \\
Adhesive & $0.0254 \mathrm{~mm}$ \\
Polyimide & $0.0508 \mathrm{~mm}$ \\
Metal 3 & $0.0180 \mathrm{~mm}$ \\
\hline Coating 2 $(0.0508 \mathrm{~mm})$ & Dimension \\
\hline Same as Coating 1 & \\
\hline Rigid 2 $(0.1524 \mathrm{~mm})$ & Dimension \\
\hline Same as Rigid 1, and Metal 4 is identical to Metal 1
\end{tabular}

Table 2 lists the detailed specification of the flex-rigid printed circuit board build-up shown in Fig. 1. It is seen in Tables 1 and 2 that the substrate height between Metal 1 and 2 and between Metal 3 and 4 is $0.1524 \mathrm{~mm}$, whereas the substrate height between Metal 2 and 3 is $0.1270 \mathrm{~mm}$.

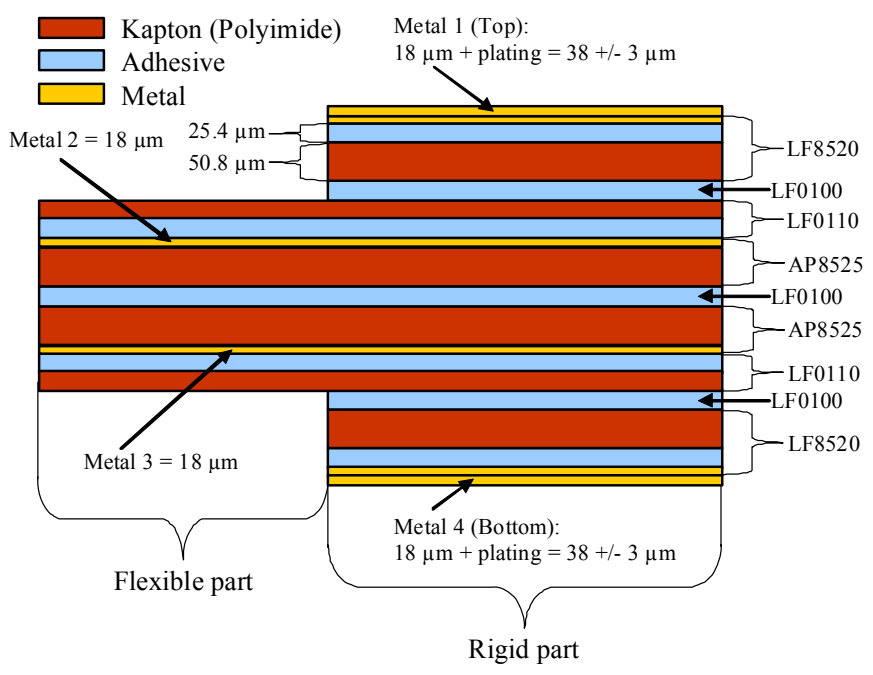

Fig. 1. Printed circuit board structure.

\section{A. Triplexer}

Fig. 2(a) shows the schematic of the proposed triplexer. The network is realized with a microstrip technology using the manifold principle of operation. The triplexer consists of three series quarter-wavelength transmission lines, three bandpass filters, and three transmission lines for tuning of the filter impedance. The principle of operation is that each sub-band has a matched input impedance ( $50 \Omega$ in this design) at the junction, but a high input impedance at the input of other subbands. The series transmission lines of three different quarter wavelengths provide a high impedance at the respective frequency band, e.g., preventing sub-band \#1 signals to reach sub-band $\# 2$ and \#3. Ideally, the series transmission provides an admittance inversion at each passband, i.e., by rotating a quarter of a wavelength from short to open. Using a short as 
the reference the series transmission lines then the ideal length is $n * \lambda / 2+\lambda / 4(n=0,1,2, .$.$) , e.g., n=0$ that gives a shortest possible line-length of $\lambda / 4$. The filter tuning lines at the junctions optimize the stop band impedance of each filter, to provide a high stop band impedance in the neighboring bands. Neighboring sub-bands are mostly critical to a sub-band since the band-pass filter has limited rejection close to its pass-band. The entire network is optimized together with the filters to achieve flat passbands and a symmetric performance between the sub-bands. Moreover, in this design a $3 / 2 \lambda(n=1$, electrical length $=270^{\circ}$ ) rotation was used to achieve a sharper cut-off. Table 3 lists the specifications of the triplexer filters, where length is the length of the filter section, width metal 1 and 2 are the widths of the layer 1 and 2 conductors in the broadside-coupled filter section, conductor width is the width of the edge-coupled filter section conductors, and spacing is the distance between the conductors. Note that the filters are symmetric, i.e., filter section 1 and 5 are equal, whereas 2 and 4 are also equal.

Table 3. Combined broadside- and edge-coupled filter specifications

\begin{tabular}{lccc}
\hline \multicolumn{1}{c}{$\begin{array}{c}\text { Filter } \\
\text { Parameter }\end{array}$} & $\begin{array}{c}\text { Sub-band } \\
\# 1\end{array}$ & $\begin{array}{c}\text { Sub-band } \\
\# 2\end{array}$ & $\begin{array}{c}\text { Sub-band } \\
\# 3\end{array}$ \\
\hline \multicolumn{3}{l}{ Broadside-coupled filter section (1 and 5) } & \\
\hline Length (mm) & 6.34 & 5.42 & 4.77 \\
Width, metal 1 (mm) & 0.70 & 0.70 & 0.70 \\
Width, metal 2 (mm) & 0.35 & 0.35 & 0.35 \\
\hline Edge-coupled filter section (2 and 4) & & \\
\hline Length (mm) & 6.34 & 5.42 & 4.80 \\
Conductor width (mm) & 0.27 & 0.27 & 0.27 \\
Spacing (mm) & 0.10 & 0.10 & 0.10 \\
\hline Edge-coupled filter section (3) & & \\
\hline Length (mm) & 6.31 & 5.39 & 4.77 \\
Conductor width (mm) & 0.28 & 0.28 & 0.28 \\
Spacing (mm) & 0.14 & 0.14 & 0.14 \\
\hline
\end{tabular}

Fig. 2(b) shows a photo of the implementation prototype. The size of the triplexer prototype is $48 \times 25 \mathrm{~mm}$. The four edge mounted Sub-Miniature A (SMA) connectors are seen. Port 1 is the input/ output (6-9 GHz), Port 2 is for sub-band \#1 (6-7 GHz), Port 3 is for sub-band \#2 (7-8 GHz), and Port 4 is for sub-band \#3 (8-9 GHz).

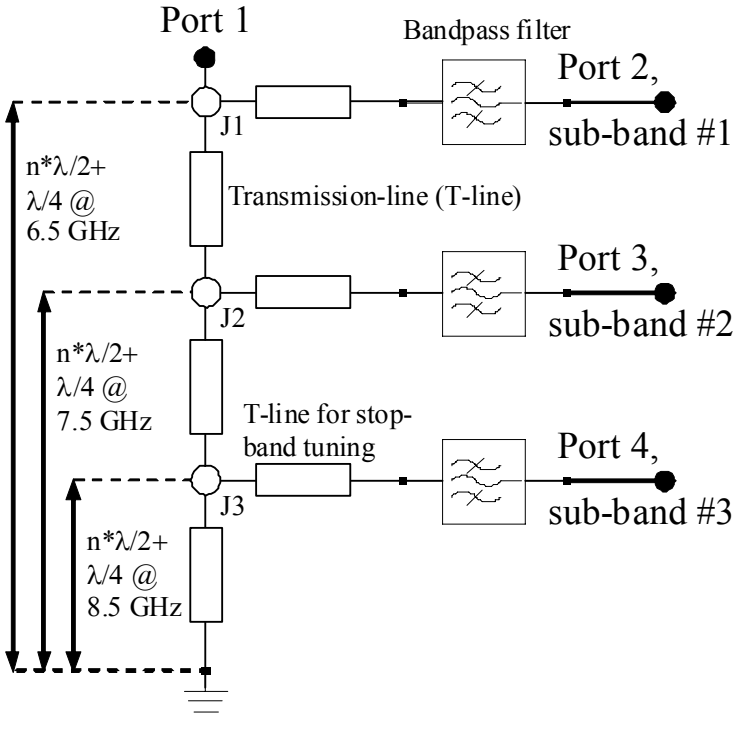

(a) Principle of the triplexer.

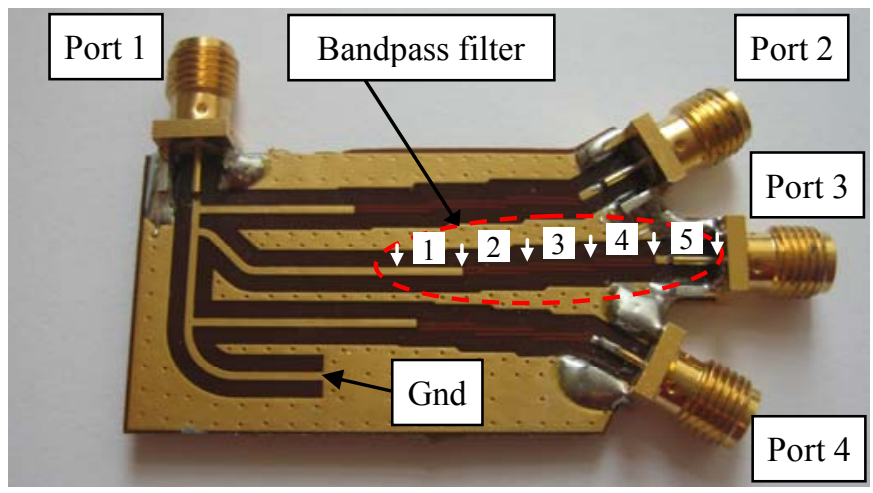

(b) Photo of the implementation.

Fig. 2. Triplexer: (a) principle of the triplexer, and (b) photo of the implementation prototype.

\section{B. Broadside- and edge-coupled filter}

Fig. 3 shows how the combined broadside- and edgecoupled filter technology is implemented in the flex-rigid printed circuit board. The start and the stop segments are placed on Metal layer 1, and the rest of the filter is placed on Metal layer 2. A bandpass filter with five coupled sections, two orders from broadside-coupling and three orders from edge-coupling, are utilized. The filter was designed for a flat passband response.

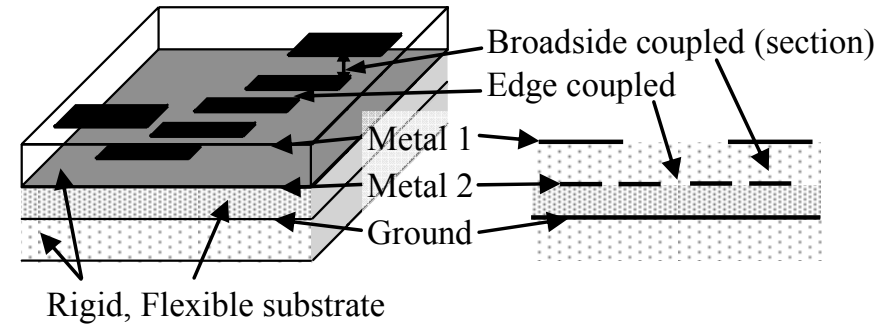

Fig. 3. Combined broadside- and edge-coupled filter. 


\section{THEORETICAL ANALYSIS}

The triplexer circuitry presented in this paper operates according to the Manifold principle [2], [3] and [12]. Equation (1)-(6) describes the principle of operation illustrated in Fig. 4 , starting at the ground reference and moving upwards along the vertical transmission-lines $\left(\mathrm{d}_{\mathrm{mn}}\right)$. From junction 3 , the ground reference is to be considered as a short-circuited stub. Where $\mathrm{Zin}_{\mathrm{JnL}}$ is the input impedance of the next stage in the manifold network, $\mathrm{Zin}_{\mathrm{JnU}}$ is the input impedance looking into junction $\mathrm{n}$, and $\mathrm{Zin}_{* \mathrm{n}}$ is the input impedance of sub-band $\mathrm{n}$ looking from the junction. Moreover, $\beta$ is the phase constant $(=2 \pi / \lambda)$, and $\mathrm{d}_{\mathrm{tn}}$ are the horizontal transmission-lines.

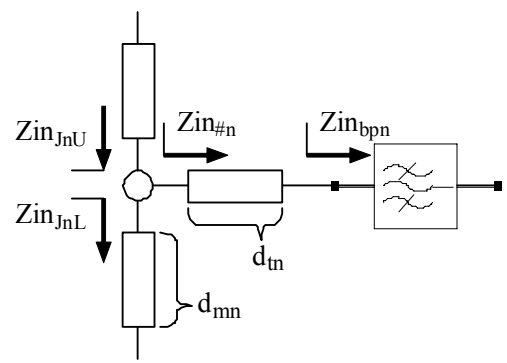

Fig. 4. A sub-band junction and associated variable definitions.

$$
\begin{aligned}
& \operatorname{Zin}_{J 3 L}=j Z_{0} \tan \left(\beta d_{m 3}\right) \\
& \operatorname{Zin}_{J 3 U}=\operatorname{Zin}_{J 3 L} / / \operatorname{Zin}_{\# 3} \\
& \operatorname{Zin}_{J 2 L}=Z_{0} \frac{\operatorname{Zin}_{J 3 U}+j Z_{0} \tan \left(\beta d_{m 2}\right)}{Z_{0}+j \operatorname{Zin}_{J 3 U} \tan \left(\beta d_{m 2}\right)} \\
& \operatorname{Zin}_{J 2 U}=\operatorname{Zin}_{J 2 L} / / \operatorname{Zin}_{\# 2} \\
& Z_{i_{J 1 L}}=Z_{0} \frac{Z_{J 2 U}+j Z_{0} \tan \left(\beta d_{m 1}\right)}{Z_{0}+j Z_{i 2 U} \tan \left(\beta d_{m 1}\right)} \\
& \operatorname{Zin}_{J 1 U}=\operatorname{Zin}_{J 1 L} / / \operatorname{Zin}_{\# 1}
\end{aligned}
$$

From the respective junction looking into each sub-band we get, where $\mathrm{Zin}_{\mathrm{Hx}}$ is the input impedance of the respective subbands and $\mathrm{Zin}_{\mathrm{bpn}}$ is input impedance of the filter at the subband.:

$$
\begin{aligned}
& Z_{i n_{\# 1}}=Z_{0} \frac{Z i n_{b p 1}+j Z_{0} \tan \left(\beta d_{t 1}\right)}{Z_{0}+j{Z i n_{b p 1}} \tan \left(\beta d_{t 1}\right)} \\
& {Z \operatorname{in}_{\# 2}}=Z_{0} \frac{\operatorname{Zin}_{b p 2}+j Z_{0} \tan \left(\beta d_{t 2}\right)}{Z_{0}+j \operatorname{Zin}_{b p 2} \tan \left(\beta d_{t 2}\right)}
\end{aligned}
$$

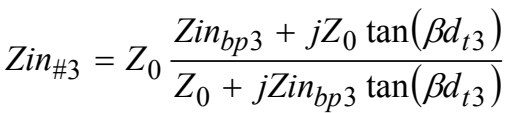

The broadside- and edge-coupled filters are built up with coupled line sections, each with unique even- and odd-mode impedances, $Z_{0 \mathrm{e}}$ and $Z_{0 \mathrm{o}}$, respectively are cascaded to create the band-pass filter. The geometrical design of each edge and broadside coupled segments determine their respective odd and even mode impedance. The combination of even and odd mode determines the characteristics of each section. Even and odd mode sections can be exchanged but due to their respective physical build-up, broadside coupled sections are suitable for sections that requires relatively strong coupling and vice versa [23]. Hence, two closely spaced metal surfaces on top of each other exhibits a strong coupling, low impedance in-between (Lower odd mode impedance compared to an edge coupled section). Fig. 3 in the previous section shows an illustration of a band-pass filter with five coupled sections. The input impedance of a section $\left(\mathrm{Zin}_{\mathrm{cl}}\right)$ is defined as [41]:

$$
\operatorname{Zin}_{c l}=\frac{1}{2 \sin (\beta l)} \sqrt{\left(Z_{0 e}-Z_{0 o}\right)^{2}-\left(Z_{0 e}+Z_{0 o}\right)^{2} \cos ^{2}(\beta l)}
$$

Where the odd- and even-mode characteristic line impedances for each section are chosen as [41]:

$$
\begin{aligned}
& \left.Z_{0 o}\right|_{n}=Z_{0}\left(1-Z_{0} J_{n}+\left(Z_{0} J_{n}\right)^{2}\right) \\
& \left.Z_{0 e}\right|_{n}=Z_{0}\left(1+Z_{0} J_{n}+\left(Z_{0} J_{n}\right)^{2}\right)
\end{aligned}
$$

The $Z_{0 \mathrm{o}}$ and $Z_{0 \mathrm{e}}$ ratio factor $\mathrm{J}_{\mathrm{n}}$ gives the frequency bandwidth and shape of the filter, where $g_{0}, g_{1}, . . g_{n}$ are standard filter coefficients. BW is the normalized relative bandwidth [41]:

$$
\begin{aligned}
& J_{1}=\frac{1}{Z_{0}} \sqrt{\frac{\pi B W}{2 g_{0} g_{1}}} \\
& J_{n}=\frac{1}{Z_{0}} \frac{\pi B W}{2 \sqrt{g_{n} g_{n+1}}} \\
& J_{N}=\frac{1}{Z_{0}} \sqrt{\frac{\pi B W}{2 g_{N} g_{N+1}}}
\end{aligned}
$$

Fig. 5(a) and (b) shows the calculated real and imaginary input impedance responses of a combined broadside- and edge-coupled bandpass filter, respectively. The filter has five coupled sections, and a passband between 6 to $7 \mathrm{GHz}$. Also note that the y-axis is in logarithmic scale. 


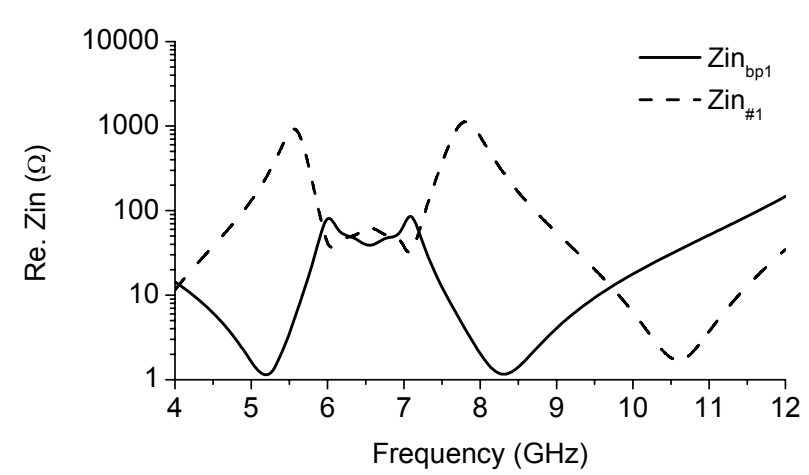

(a)

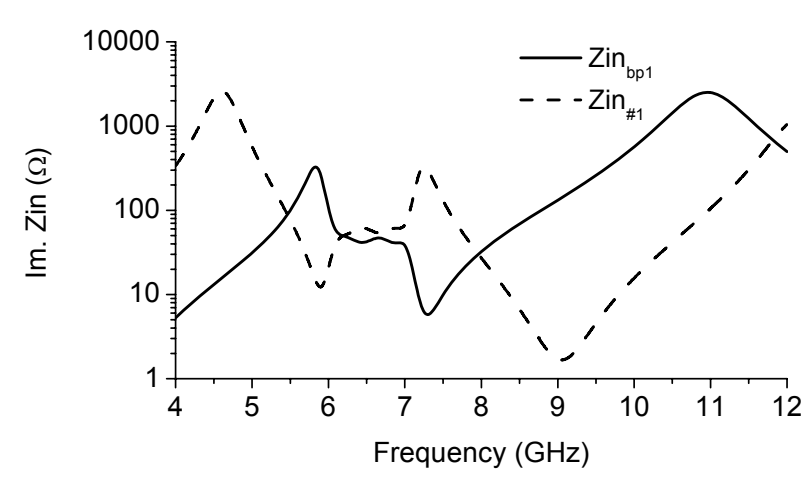

(b)

Fig. 5. 6-7 GHz band-pass filter, input impedance: (a) real values, and (b) imaginary values.

The vertical transmission-lines in the manifold provide a high input impedance towards the next manifold stage $\left(\mathrm{Zin}_{\mathrm{JnL}}\right)$ in the passband of the sub-band at the respective junction. Similarly, the horizontal "T-line for stop-band tuning" is to provide a high sub-band input impedance $\left(\mathrm{Zin}_{* n}\right)$ in the stopband frequencies close to the sub-band. The solid curves in Fig. 5(a) and (b) show typical input impedance responses of a coupled line filter. It is shown that the stop-band near the passband is close to short, i.e., a direct connect to the junction would severely affect the neighboring sub-bands according to equation (2)-(6). The dashed curve shows the input impedance when a microstrip-line with the length $\left(\mathrm{d}_{12}\right)$ is added. With the additional electrical length the stop-band impedance response is rotated to a high value, i.e., approximately equal to an electrical open in neighboring passband frequencies.

Fig. 6 shows how forward transmission of a sub-band (subband $\# 1$ in this example) and the common port input impedance is affected by the stop-band impedances of neighboring sub-bands. The calculations in Fig. 6a are performed using the following assumptions: the total vertical transmission line $\left(\mathrm{d}_{\mathrm{m} 1}+\mathrm{d}_{\mathrm{m} 2}+\mathrm{d}_{\mathrm{m} 3}\right)$ is assumed to be $\lambda / 4, \mathrm{Zin}_{\# 3}>>$ $\mathrm{Zin}_{\# 1}$, and $\mathrm{Z}_{0}=50 \Omega$. Furthermore, the filters and all transmission lines are assumed to be loss-less. It is seen that a low input impedance of a neighboring sub-band has severe impact on the multiplexer performance, and when the stop band impedance increases the impact reduces quickly. The calculations shown in Fig. 6(b) were done using the same assumptions as in 6(a), but it also includes the impact of nonideal $\mathrm{Zin}_{\# 3}$ (Impact of sub-band \#3 on sub-band \#1:s forward transmission). Similarly as in Fig. 6(a) it is seen that when the stop-band impedance increases the impact reduces quickly. Moreover, note that the lines when $\mathrm{Zin}_{\sharp 3}=600,800$, and 1000 $\Omega$ are hard to distinguish due to the small difference.

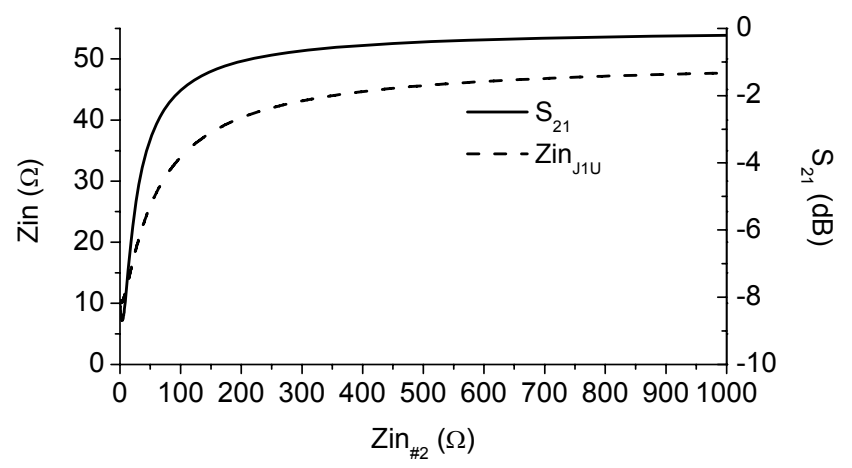

(a)

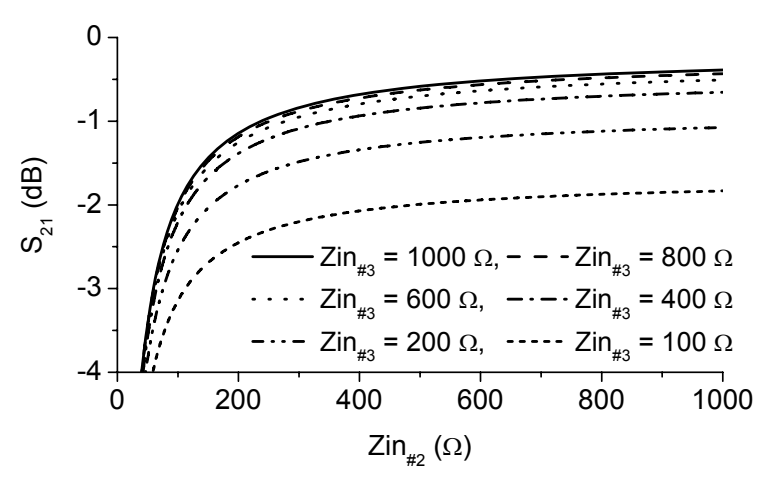

(b)

Fig. 6. Forward transmission and input impedance: (a) $\mathrm{S}_{21}$ and $\mathrm{Zin}_{\mathrm{JIU}} \mathrm{vs} \mathrm{Zin}_{\# 2}$, and (b) $S_{21}$ vs Zin ${ }_{* 2}$ at different $Z_{\text {in }}{ }_{* 3}$.

\section{Simulated And Measured Results}

Design and simulation were done with Advanced Design System (ADS) 2009 from Agilent technologies Inc. Measurements were done with a Rhode\&Schwartz ZVM vector network analyzer.

\section{A. Triplexer}

Fig. 7(a) shows the simulation and measurement results of forward transmission of the triplexer. A flat, ripple-free passband response is seen. The transmission line network is optimized together with the filters to achieve high blocking of neighbouring bands. A good match between simulation and measurement results is seen. The complete flex-rigid multilayer printed circuit board was simulated with radiation characteristics enabled in ADS for the best accuracy. However, some limitations still exist in the simulations. The SMA connectors are not included, and ADS Momentum does not take surface roughness into count. The simulated insertion loss is $3.35-3.85 \mathrm{~dB}$, and measured insertion loss is $3.41-4.10$ $\mathrm{dB}$ for the three sub-bands. As a comparison it is worth mentioning that simulation of stand alone filters indicated an 
insertion loss between 2.34-2.49 dB (Only filter). All subbands have at least $1 \mathrm{GHz}$ bandwidth at $-3 \mathrm{~dB}$ from the top. Fig. 7(b) and (c) show the simulation and measurement results of return loss of the triplexer expressed with S-parameters. Smooth forward transmission and neighbouring band blocking were prioritized over low return loss, as seen in Fig. 7(a)-(c). The only really noticeable difference is a small frequency shift, due to the fact that the simulated phase velocity differs from the actual, which is a known fact for this substrate stack [4], [23] and [36]. Because of this well known difference a scaled version of the triplexer was also designed, fabricated and measured, see the dotted line labelled $-5 \%$ in Fig. 7(a). The $-5 \%$ notation is with respect to target frequency, i.e., all electrical lengths were scaled up with $5 \%$. Note that the $-5 \%$ (dotted lines) overlap a lot with the simulated results (solid lines). The design freedom to achieve a low return loss and smooth forward transmission is therefore limited. Comparing simulated and measured input reflection it is seen that the curves have the same shape but some narrowband variations exist. This is likely due to the fact that the SMA connectors were not included in the simulations [23]. Fig. 7(d) and (e) show isolation between Ports 2-4. It is seen that the minimum isolation is $15 \mathrm{~dB}$. The minimum isolation occurs at the boundary of the neighbouring sub-bands, so in the three passbands the isolation is better than $15 \mathrm{~dB}$. The isolation between the non-neighbouring alternate sub-bands is $45 \mathrm{~dB}$.
Fig. 7(f) and (g) show simulated and measured phase response, respectively. It is seen that all three sub-bands have a high degree of linearity within their respective passbands. Fig. 7(h) and (i) show simulated and measured group delay, respectively. It is seen that for all the $1 \mathrm{GHz}$ sub-bands the group delay is around $1.0 \mathrm{~ns}$ and the variation is approximately $0.4 \mathrm{~ns}$ within each sub-band. It is the filter type and filter order that dominate the delay [4] and [23].

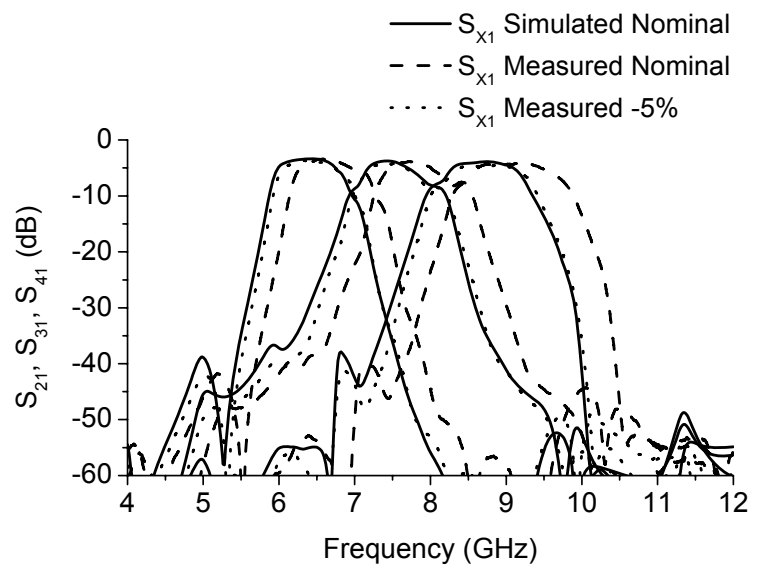

(a)

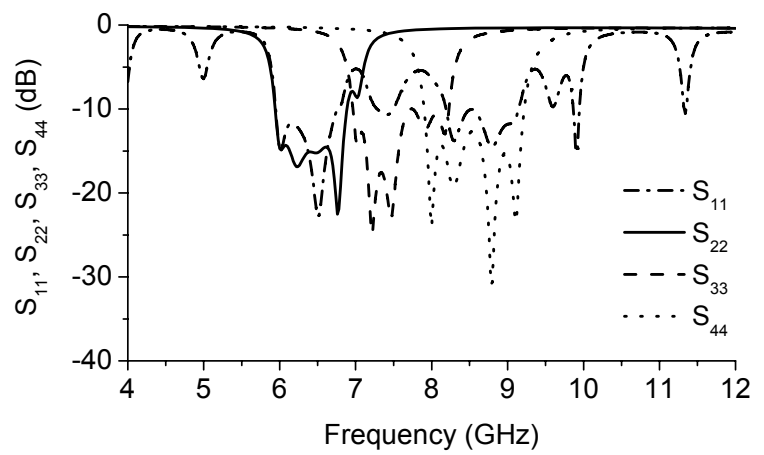

(b)

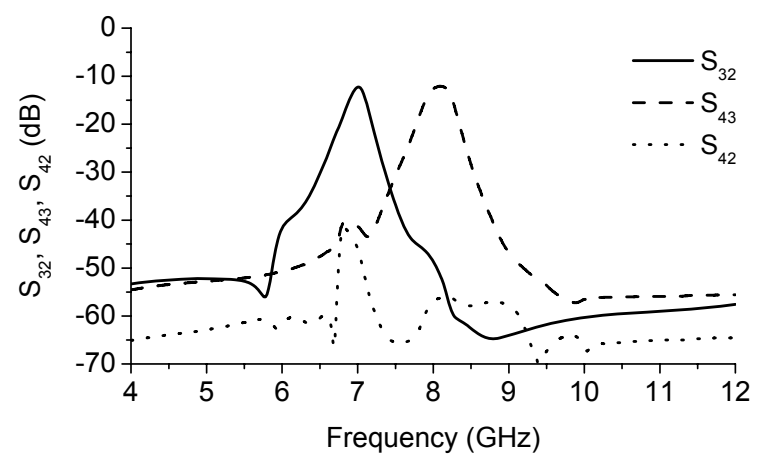

(d)

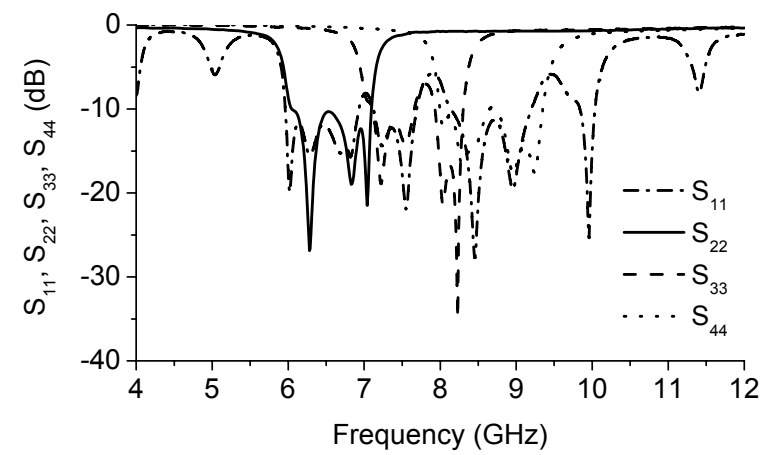

(c)

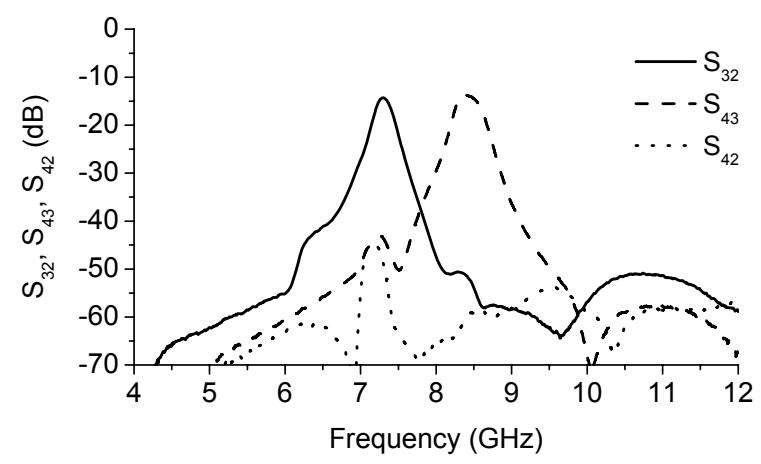

(e) 


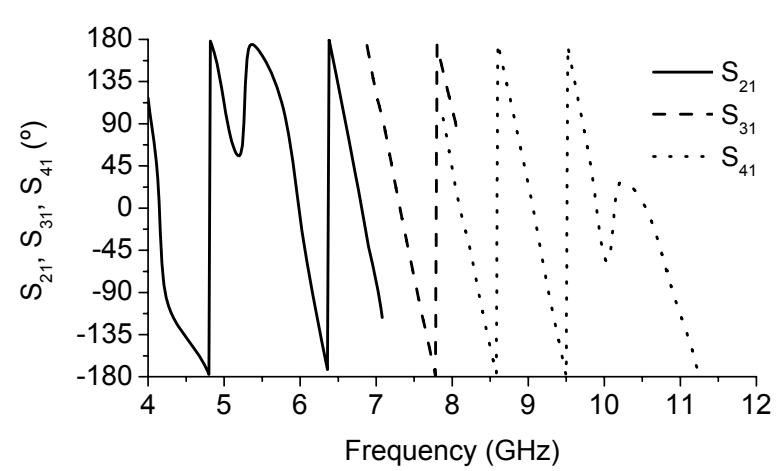

(f)

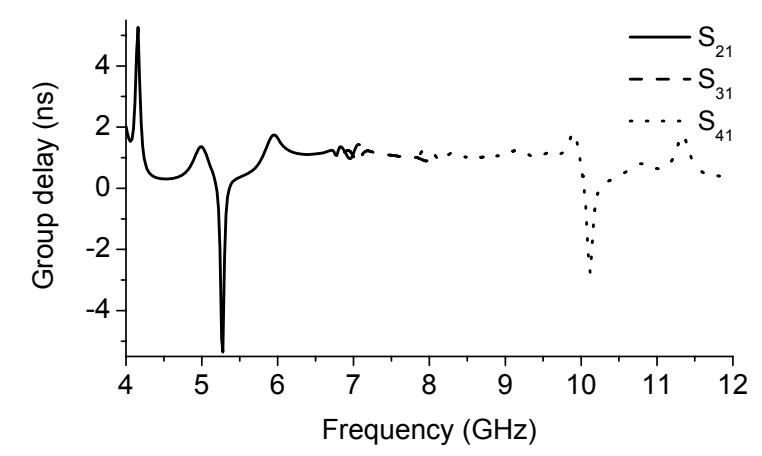

(h)

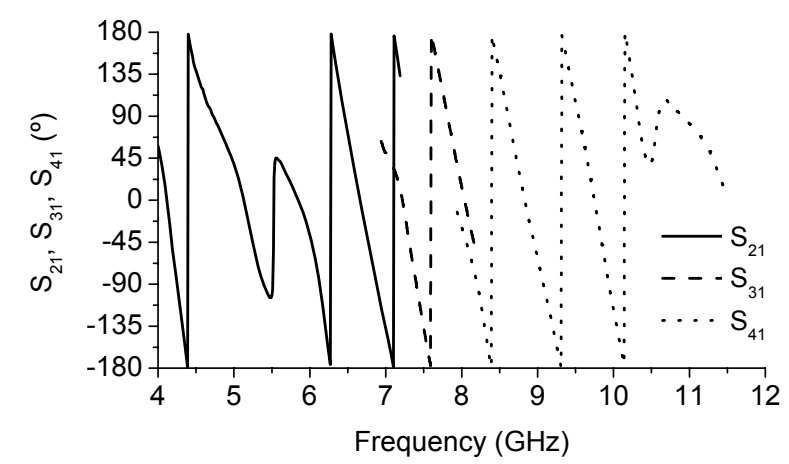

(g)

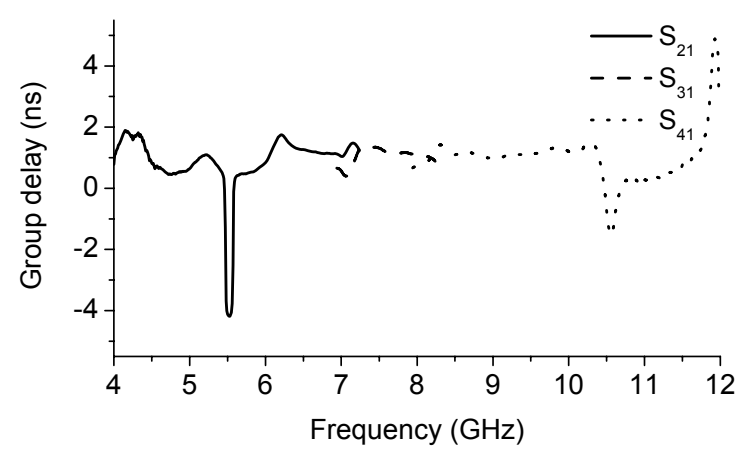

(i)

Fig. 7. Triplexer simulations and measurements: (a) simulated and measured forward transmission with the passbands marked at $-3 \mathrm{~dB}$, (b) simulated return loss, (c) measured return loss, (d) simulated isolation, (e) measured isolation, (f) simulated phase response, (g) measured phase response, (h) simulated group delay, and (i) measured group delay.

\section{DISCUSSION}

A good agreement with only minor deviations was observed between simulated and measured results. For instance, measured insertion loss was approximately $0.2 \mathrm{~dB}$ more than the simulated one. A slightly upward shift in frequency is also seen, i.e., a constant error of $5 \%$ which is typical known deviation for this substrate [37]. The frequency shift is due to the fact that the simulated electrical length of the distributed circuitry differs from the measured one, i.e., the simulated phase velocity is lower than the measured one. This is proven by the additional "-5\%" scaled design, see in Fig. 7(a). The higher insertion loss from the measurements can be explained by the surface roughness that is not included in the simulations because Momentum in ADS does not support that. It is a known fact that surface roughness increases insertion loss [23], [42], [43]. Actually surface roughness could in the worst case add even more than the difference seen here, roughly up to half a $\mathrm{dB}$ at the line-lengths used here at 6-9 $\mathrm{GHz}$ [23]. Moreover, some additional insertion-loss may come from reflections caused by the SMA connectors soldered on the triplexer [23].

The group delay variation was measured to be about $0.4 \mathrm{~ns}$ within each sub-band. How this impact on a system performance depends of the implementation and a simple conclusion cannot be directly drawn. However, in pulsed systems a small group delay variation might already cause degradation of system performance, while in orthogonal frequency division multiplexing systems group delay impact on the system level performance is limited [28].

By using broadside coupled microstrips, the printed circuit board tolerance requirement on the minimum spacing for the whole triplexer is relieved. Thus, this triplexer can be integrated into a flex-rigid four metal layer printed circuit board without any particular requirements on the fabrication process. This opens way for full integration of the radio frontend on flex-rigid printed circuit boards, including a flexible antenna in the future. Hence, compared to previously known works the flex rigid printed circuit board technology can host distributed circuitry with similar performance as conventional high frequency printed circuit boards [14], [18], [23], [44].

\section{CONCLUSION}

A fully printed circuit board integrated planar triplexer using microstrips for three UWB bands within the 6-9 GHz frequency band has been studied and presented. The theoretical analysis shows that high stop-band input impedance in adjacent channels is crucial for a high performance (e.g., low insertion loss) when utilizing the manifold multiplexing principle. A demonstrator on a flexrigid printed circuit board for the 6-9 GHz frequency band was designed and evaluated. Three flat 1-GHz sub-bands have been achieved. The triplexer can be integrated into a multilayer flex-rigid printed circuit board using a commercial process technique at low cost, even though the sub-bands are 
side by side without any guard-band in between. A phase response with a high degree of linearity and a small group delay variation of $0.4 \mathrm{~ns}$ are observed for all the three subbands. Finally, detailed modeling of the flexible, rigid, and adhesion layers provided good simulation prediction of the prototype over all performance, e.g., see Fig. 1 and 7(a).

\section{REFERENCES}

[1] F. S. Coale, "Applications of Directional Filters for Multiplexing Systems," Trans. Microwave Theory and Tech., vol. 6, no. 4, pp. 450453, Oct. 1958

[2] J. D. Rhodes and R. Levy, "A generalized multiplexer theory," Trans. Microwave Theory and Tech., vol. 27, no. 2, pp. 111-123, Feb. 1979.

[3] J. D. Rhodes and R. Levy, "Design of General Manifold Multiplexers," Trans. Microwave Theory and Tech., vol. 27, no. 2, pp. 111-123, Feb. 1979.

[4] J. W. Bandler, W. Kellermann, and K. Madsen, "A nonlinear $1_{1}$ optimization algorithm for design, modeling, and diagnosis of networks," IEEE Trans. Circuits and Systems, vol. 34, no. 2, pp. 174181, Feb. 1987

[5] R. R. Mansour, "Design of Superconductive Multiplexers Using SingleMode and Dual-Mode Filters," Trans. Microwave Theory and Tech., vol. 41, no. 7, pp. 1411-1418, Jul. 1994.

[6] C. Rauscher, "Efficient design methodology for microwave frequency multiplexers using infinite-array prototype circuits," Trans. Microwave Theory and Tech., vol. 42, no. 7, pp. 1337-1346, Jul. 1994.

[7] A. A. Kirilenko, S. L. Senkevich, V. I. Tkachenko, and B. G. Tysik, "Waveguide duplexer and multiplexer design," Trans. Microwave Theory and Tech., vol. 42, no. 7, pp. 1393-1396, Jul. 1994.

[8] G. L. Matthaei, S. M. Rohlfing, and R. J. Forse, "Design of HTS, lumped-element, manifold-type microwave multiplexers," Trans. Microwave Theory and Tech., vol. 44, no. 7, pp. 1313-1321, Jul. 1996.

[9] D. Bariant, S. Bila, D. Baillargeat, S. Verdeyme, P. Guillon, D. Pacaud, and J. J. Herren, "Method of spurious mode compensation applied to manifold multiplexer design," in IEEE MTT-S Int. Microwave Symp. Dig., 2002, vol. 3, pp. 1461-1464.

[10] T. Ohno, K. Wada, and O. Hashimoto, "Design methodologies of planar duplexers and triplexers by manipulating attenuation poles," IEEE Trans. Microwave Theory and Tech., vol. 53, no. 6, pp. 2088-2095, Jun. 2005.

[11] C.-F. Chen, T.-Y. Huang, C.-P. Chou, and R.-B. Wu, "Microstrip duplexers design with common resonator sections for compact size, but high isolation," Trans. Microwave Theory and Tech., vol. 54, no. 5, pp. 1945-1952, May 2006.

[12] R. I. Cameron; M. Yu, "Design of manifold-coupled multiplexers," Microwave Magazine, IEEE, vol. 8, no. 5, pp. 46-59, Oct. 2007.

[13] M.-I Lai; S.-K. Jeng, "A microstrip three-port and four-channel multiplexer for WLAN and UWB coexistence," Trans. Microwave Theory and Tech., vol. 53, no. 10, pp. 3244-3250, Oct. 2005.

[14] S. Mallegol; J.-P. Coupez, C. Person, T. Lespagnol, S. Paquelet; A. Bisiaux, "Microwave (De)Multiplexer for Ultra-Wideband (UWB) NonCoherent High Data Rates Transceiver," in Proc. 3rd European Radar Conference, EuRAD 2006, pp. 346-349.

[15] K. Stadius; T. Rapinoja; J. Kaukovuori; J. Ryynanen; and K. A. I. Halonen, "Multitone Fast Frequency-Hopping Synthesizer for UWB Radio," Trans. Microwave Theory and Tech., vol. 55, no. 8, pp.16331641, Aug. 2007

[16] S.-H. Tarng, Y.-C. Tsai; Y.-S. Shen; C. F. Jou, "A Signal Generator for MB-OFDM UWB System in $0.18 \mu \mathrm{m}$ CMOS Process," in IEEE MTT-S Int. Microwave Symp., 2007, pp. 2157-2160.

[17] C. Galbraith; G. M. Rebeiz; R. Drangmeister, "A Cochlea-based Preselector for UWB Applications," in IEEE Radio Frequency Integrated Circuits (RFIC) Symp., 2007, pp. 219-222.

[18] S.-J. Zeng, J.-Y. Wu, and W.-H. Tu "Compact and High-Isolation Quadruplexer Using Distributed Coupling Technique," IEEE Microwave and Wireless Comp. Lett., vol. 21, no. 4, Apr. 2011.

[19] M. Yu, and Y. Wang, "Enhanced Microwave Multiplexing Network," Trans. Microwave Theory and Tech., vol. 59, no. 2, pp. 270-277, Feb 2011.
[20] M. Karlsson, P. Håkansson, A. Huynh, and S. Gong, "Frequencymultiplexed Inverted-F Antennas for Multi-band UWB," in Proc. IEEE Wireless and Microwave Conf., 2006, pp. 2.1-2.3.

[21] A. Serban, M. Karlsson, and S. Gong, "All-Microstrip Design of Three Multiplexed Antennas and LNA for UWB Systems," in Proc. AsiaPacific Microwave Conf., 2006, pp. 1109-1112.

[22] P. Håkansson, A. Huynh, and S. Gong, "A Study of Wireless Parallel Data Transmission of Extremely High Data Rate up to 6.17 Gbps per Channel," in Proc. Asia-Pacific Microwave Conf., 2006, pp. 975-979.

[23] M. Karlsson, P. Hakansson, S. Gong, "A Frequency Triplexer for UltraWideband Systems Utilizing Combined Broadside- and Edge-Coupled Filters," IEEE Trans. on Advanced Packaging, vol. 31, no. 4, pp. 794801, Nov. 2008

[24] M. Karlsson, A. Huynh, S. Gong, "Parallel channels using frequency multiplexing techniques," In: Ultra Wideband, SCIYO; 2010. p. 35-54.

[25] "First report order, revision of part 15 of commission's rules regarding ultra-wideband transmission systems" FCC, Washington, 2002.

[26] G. R. Aiello and G. D. Rogerson, "Ultra Wideband Wireless Systems," IEEE Microwave Magazine, vol. 4, no. 2, pp. 36-47, Jun. 2003.

[27] L. Yang and G. B. Giannakis, "Ultra-Wideband Communications, an Idea Whose Time has Come," IEEE Signal Processing Magazine, pp. 26-54, Nov. 2004

[28] J. Balakrishnan, A. Batra, and A. Dabak, "A multi-band OFDM system for UWB communication," Proc. Conf. Ultra-Wideband Systems and Technologies, Reston, VA, 2003, pp. 354-358.

[29] W. D. Jones, "Ultrawide gap on ultrawideband," IEEE Spectrum, vol. 41, no. 1, pp. 30, Jan. 2004.

[30] D. Geer, "UWB standardization effort ends in controversy," Computer, vol. 39 , no. 7, pp. 13-16, Jul. 2006.

[31] W. Hirt, "The European UWB Radio Regulatory and Standards Framework: Overview and Implications," IEEE International Conference on Ultra-Wideband, ICUWB 2007, pp.733-738, 24-26 Sept. 2007

[32] A. Giorgetti, M. Chiani, D. Dardari, R. Piesiewicz, G. H. Bruck, "The Cognitive Radio paradigm for Ultra-Wideband systems: The European Project EUWB," IEEE International Conference on Ultra-Wideband, ICUWB 2008, vol.2, pp.169-172, 10-12 Sept. 2008.

[33] S. Chakraborty, N. R. Belk, A. Batra, M. Goel, and A. Dabak, "Towards fully integrated wideband transceivers: fundamental challenges, solutions and future," Proc. IEEE Radio-Frequency Integration Technology: Integrated Circuits for Wideband Communication and Wireless Sensor Networks 2005. pp. 26-29, 2 Dec. 2005.

[34] MultiBand OFDM Alliance SIG, "MultiBand OFDM Pysical Layer Proposal for IEEE 802.15 Task Group 3a," Sep. 2004.

[35] K. Li, D. Kurita, T. Matsui, "An ultrawideband bandpass filter using broadside-coupled microstrip-coplanar waveguide structure" in IEEE MTT-S Int. Microwave Symp. Dig., 2005, pp. 675-678.

[36] P.-H. Deng, C.-H. Wang, C. H. Chen, "Novel Broadside-Coupled Bandpass Filters Using Both Microstrip and Coplanar-Waveguide Resonators", Trans. Microwave Theory and Tech., vol. 54, no. 10, pp. 3746-3750, Oct. 2006.

[37] R. Dokania, X. Wang, S. Tallur, C. Dorta-Quinones, A. Apsel, "An Ultralow-Power Dual-Band UWB Impulse Radio," IEEE Trans. on Circuits and Systems II: Express Briefs, vol. 57, no. 7, pp. 541-545, July 2010.

[38] S. Sen, F. Aryanfar, C. Werner, "A Multiband Transceiver System in 45nm CMOS for Extended Data Rate through Notchy Wireline Channels," IEEE Trans. on Circuits and Systems II: Express Briefs, vol. 58, no. 9, pp. 545-549, Sept. 2011.

[39] H. Darabi, A. Mirzaei, M. Mikhemar, "Highly Integrated and Tunable RF Front Ends for Reconfigurable Multiband Transceivers: A Tutorial," IEEE Trans. on Circuits and Systems I: Regular Papers, vol. 58, no. 9, pp. 2038-2050, Sept. 2011.

[40] M. Karlsson, and S. Gong, "Circular Dipole Antenna for Mode 1 UWB Radio with Integrated Balun Utilizing a Flex-rigid Structure," IEEE Trans. Antennas and Propag., vol. 57, no. 10, pp. 2967-2971, Oct. 2009.

[41] R. Ludwig and G. Bogdanov, "RF Circuit Design - Theory and Application," 2nd ed., Upper Saddle River, New Jersey, Pearson Education Inc., 2005.

[42] X. Gu; L. Tsang; H. Braunisch, "Estimation of Roughness-Induced Power Absorption From Measured Surface Profile Data," Microwave and Wireless Components Lett., IEEE, vol. 17, no. 7, pp. 486-488, Jul. 2007. 
[43] X. Chen, "EM Modeling of Microstrip Conductor Losses Including Surface Roughness Effect," Microwave and Wireless Components Lett., IEEE, vol. 17, no. 2, pp. 94-96, Feb. 2007.

[44] A. Al. Tanany, D. Gruner, F. Goelden, K. Bathich, R. Kahl, G. Boeck, "Efficiency improvement for RF power amplifiers by employing harmonic injection at the input," Microwave Conference (GeMIC), 2011 German, pp.1-4, 14-16 Mar. 2011.

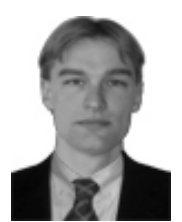

Magnus Karlsson was born in Västervik, Sweden in 1977. He received his M.Sc., Licentiate of Engineering and Ph.D. degrees from Linköping University in Sweden, in 2002, 2005 and 2008, respectively.

In 2003 he started in the Communication Electronics research group at Linköping University and is currently working as a senior researcher. His main work involves wideband antenna-techniques, wideband transceiver front-ends, and wireless communications.

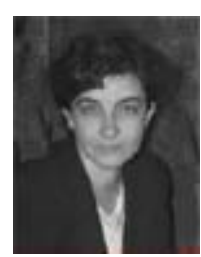

Adriana Serban received an M.Sc degree in electronic engineering from Politehnica University, Bucharest, Romania. From 1981 to 1990 she was with Microelectronica Institute, Bucharest as a Principal Engineer and was involved in mixed integrated circuit design. From 1992 to 2002 she was with Siemens AG, Munich, Germany and with Sicon AB, Linköping, Sweden as analog and mixed signal integrated circuit Senior Design Engineer. Since 2002 she has been a Lecturer at Linköping University teaching in analog/digital system design and $\mathrm{RF}$ circuit design. She is recived her Ph.D degree in Communication Electronics 2010. Her main research interest has been RF circuit design and high-speed integrated circuit design.

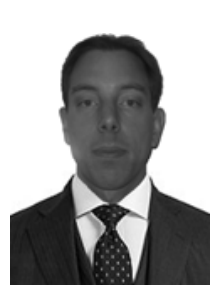

Joakim Östh received his M.Sc. degree in 2006 from Linköping University, Sweden. Since 2009 he has been a Ph.D. degree in communication electronics at Linköping University, Sweden. His main research interest has been communication electronics including RF design, wireless communications and high-speed data transmissions.

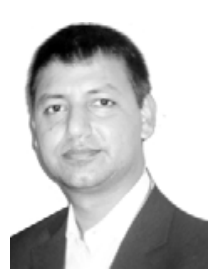

Owais received his B.E. degree in 1996 and Master degree in 2000 from University of Engineering and Technology Peshawar Pakistan. $\mathrm{He}$ is employed as assistant professor at Comsats Institute of Information Technology, Abbotabad, Pakistan. Since 2007 he has been PhD student in communication electronics at Linköping University, Sweden. His main research interest has been Antenna design and high-speed data transmissions.

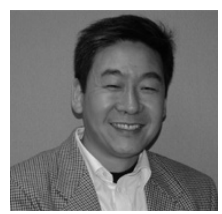

Shaofang Gong was born in Shanghai, China, in 1960. He received his B.Sc. degree from Fudan University in Shanghai in 1982, and the Licentiate of Engineering and Ph.D. degrees from Linköping University in Sweden, in 1988 and 1990, respectively.

Between 1991 and 1999 he was a senior researcher at the microelectronic institute - Acreo in Sweden. From 2000 to 2001 he was the CTO at a spin-off company from the institute. Since 2002 he has been full professor in communication electronics at Linköping University, Sweden. His main research interest has been communication electronics including RF design, wireless communications and high-speed data transmissions. 